(1)

CrossMark

\title{
Don't forget about neuromuscular disorders!
}

\author{
Wolfram Windisch
}

Affiliation: Cologne Merheim Hospital, Dept of Pneumology, Kliniken der Stadt Köln, gGmbH, Witten/ Herdecke University, Faculty of Health/School of Medicine, Köln, Germany.

Correspondence: Wolfram Windisch, Dept of Pneumology, Cologne Merheim Hospital, Kliniken der Stadt Köln $\mathrm{gGmbH}$, Witten/Herdecke University, Faculty of Health/School of Medicine, Ostmerheimer Strasse 200, D-51109 Köln, Germany. E-mail: windischwakliniken-koeln.de

@ERSpublications

In patients with NMD, ineffective coughing, recurrent aspiration and airway infections, respiratory failure and home mechanical ventilation require the expertise of respiratory physicians. Therefore, we should not forget about neuromuscular disorders! http://ow.ly/UeEA30lFMxn

Cite this article as: Windisch W. Don't forget about neuromuscular disorders! Eur Respir J 2018; 52: 1801657 [https://doi.org/10.1183/13993003.01657-2018].

Respiratory medicine ostensibly targets diseases of the airways and the lungs, while respiratory problems arising from other entities are suggested to only play a minor role in this branch of medicine. However, diseases of the respiratory pump, particularly those associated with neuromuscular disorders (NMD), bear significant relevance to respiratory physicians, both in clinical practice and in terms of the scientific evolution of respiratory medicine and mechanical ventilation $[1,2]$. During the 1952 Copenhagen polio epidemic, polio victims with severe respiratory acidosis secondary to respiratory paralysis were the first group of patients in whom mortality could be dramatically reduced by applying positive pressure ventilation in combination with careful airway management [3]. As things developed, the protagonist of this accomplishment and hero of the Copenhagen polio epidemic, Bjørn Ibsen, became known to many as the founding father of modern intensive care medicine [4].

Nowadays, it is not just patients with post-polio sequelae, but also all other patients with NMD potentially affecting the respiratory muscles, that are well-accepted candidates for home mechanical ventilation (HMV) in the event of chronic hypercapnic respiratory failure $[1,2]$. According to the EUROVENT trial, a large epidemiological study on the patterns of HMV use in Europe, about one third of all HMV patients received HMV therapy primarily due to NMD [5]. These patients need to be regularly screened in advance to determine whether there is an indication for respiratory failure and, following the establishment of HMV, should also be regularly monitored to ascertain therapy effectiveness $[1,2]$. Moreover, ineffective coughing, recurrent aspiration, and airway infections also require the expertise of respiratory physicians. Therefore, the relevance of NMD in respiratory medicine cannot be disputed.

In this issue of the European Respiratory Journal, Rose et al. [6] report on an immense retrospective population-based longitudinal cohort study of adult NMD patients aged from 18 to 105 years. The study used a battery of different but linked health administrative databases for the Canadian province of Ontario, which harbours approximately 13 million people and covered a period between April 1, 2003 and March 31,2015. The primary aim of this study was to evaluate the use of healthcare resources by NMD patients. For this purpose, a thoughtfully devised cohort was created based on database codes covering a period of 5 years (until March 31, 2008), and this cohort was tracked for a minimum of 7 
years (until March 31, 2015). An enormous cohort comprising 185585 adult NMD patients was covered (mean age 52 years, $59 \%$ female).

The main findings were that more than one third of all NMD patients received regular respiratory specialist care, and that there was substantial emergent healthcare utilisation. Accordingly, 41173 (22\%) patients visited an emergency department for respiratory conditions at an average of 1.6 times every 3 years, and 14947 patients (8\%) were hospitalised for respiratory reasons 1.4 times every 3 years. In addition, 157285 (85\%) individuals went to the emergency department for a combined total of 1202800 visits (all-cause), while 100052 (54\%) individuals (301064 admissions overall) were admitted to hospital for a median (interquartile range) duration of 9 (3-28) days. It was concluded from these data that the burden of NMD on the public healthcare system is considerable, and that all-cause public health service utilisation for individuals with NMD is substantial [6].

There are additional important things to learn beyond these main findings: firstly, the group of patients was highly heterogeneous (per definition) with respect to the underlying diagnosis, where most of the patients $(n=94739)$ had disorders that were not further defined beyond the classical diagnostic groups [6]. Respiratory physicians should be aware that the main clinical characteristics of the many different disorders may differ considerably, and this is particularly true for the severity of respiratory muscle paralysis, the presence of cardiomyopathy, the potential for developing rhabdomyolysis during anaesthesia or intensive care therapy, and the onset of respiratory failure [2]. This further underlines the importance of NMD in respiratory medicine.

Secondly, it is quite astonishing to see that only 1446 patients eventually received HMV, while 7737 received continuous positive airway pressure and 4750 home oxygen therapy [6]. Amongst all patients, $11.9 \%$ or $15.5 \%$ had comorbid chronic obstructive pulmonary disease (COPD) or asthma, respectively, and home oxygen therapy was primarily attributed to these comorbidities. This indicates a significant overlap between NMD and pulmonary diseases. Therefore, HMV does indeed only reflect the tip of the iceberg of respiratory involvement, and respiratory problems in NMD patients may differ considerably. Indeed, detecting the extent and variety of respiratory involvement in patients with NMD needs to be stressed.

Thirdly, visits from an outpatient respiratory specialist reportedly occurred for 64084 patients (35\%) at an overall frequency of four visits every 3 years, which was reportedly on par with professional guideline recommendations [6]. However, it was also acknowledged that there was a substantial variation in visitation frequency, whereby $65 \%$ of the patients did not receive any visits from an outpatient respiratory specialist. In addition, out of all the patients presenting in the emergency department for respiratory conditions, only $9 \%$ underwent a consultation with an in-patient respiratory specialist. This indicates that there may be a significant lack of specific respiratory treatment and support resources, at least for some NMD patients. This is an important issue which needs to be verified in the future.

HMV is now known to be unequivocally associated with improvements in health-related quality of life and long-term survival [2]. Despite successful HMV, however, the prognosis of many of the diseases is substantially impaired, thus prompting the question of how far we can go with intensive care unit (ICU) medicine in NMD patients. In the study by Rose et al. [6], 29453 patients (15.9\%) required ICU admission, with an average of 1.8 admissions per year (all-cause) [6]. This is thought to be substantial. The renowned physicist Stephen Hawking, who suffered from juvenile amyotrophic lateral sclerosis, once described how there was little hope for his survival when he contracted pneumonia in 1985, and the treating physicians had even given his wife the option of turning off the ventilator. She ultimately refused, and Stephen Hawking led a fulfilling life with invasive HMV until March 2018 - what luck!

On the other hand, we have learned from recent trials that chronically tracheostomised/ventilated patients who have unsuccessfully undergone prolonged weaning after ICU treatment may have severely impaired health-related quality of life, with some patients even having expressed their wish to die rather than to continue invasive HMV [7, 8]. This held true not only for patients with COPD, but also for those with NMD. Thus, we have to face important ethical considerations when treating NMD patients with disease-related respiratory problems, and, most importantly, respiratory failure. Obviously there is no run-of-the-mill solution available for this problem. In contrast, advanced disease care in NMD requires respiratory specialists to individualise treatment approaches in accordance with the wishes of the patients and their families. The study by Rose et al. [6] is elegant and important. It reminds us that NMD patients form an important group of patients in respiratory medicine. Therefore: don't forget about neuromuscular disorders! 
Conflict of interest: W. Windisch reports that the Cologne study group received an open research grant from Weinmann/Germany, Vivisol/Germany, Heinen und Löwenstein/Germany and VitalAire/Germany, during the conduct of the study; and personal fees for speaking from companies dealing with mechanical ventilation, outside the submitted work.

\section{References}

1 Windisch W, Geiseler J, Simon K, et al. German National Guideline for Treating Chronic Respiratory Failure with Invasive and Non-Invasive Ventilation: Revised Edition 2017 - Part 1. Respiration 2018; 96: 66-97.

2 Windisch W, Geiseler J, Simon K, et al. German National Guideline for Treating Chronic Respiratory Failure with Invasive and Non-Invasive Ventilation - Revised Edition 2017: Part 2. Respiration 2018; 96: 171-203.

3 Ibsen B. The anaesthetist's viewpoint on the treatment of respiratory complications in poliomyelitis during the epidemic in Copenhagen, 1952. Proc R Soc Med 1954; 47: 72-74.

4 Reisner-Sénélar L. The birth of intensive care medicine: Björn Ibsen's records. Intensive Care Med 2011; 37: 1084-1086.

5 Lloyd-Owen SJ, Donaldson GC, Ambrosino N, et al. Patterns of home mechanical ventilation use in Europe: results from the Eurovent survey. Eur Respir J 2005; 25: 1025-1031.

6 Rose L, McKim D, Leasa D, et al. Patterns of healthcare utilisation for respiratory complications of adults with neuromuscular disease: a population study. Eur Respir J 2018; 52: 1800754.

7 Huttmann SE, Windisch W, Storre JH. Invasive home mechanical ventilation: living conditions and health-related quality of life. Respiration 2015; 89: 312-321.

8 Huttmann SE, Magnet FS, Karagiannidis C, et al. Quality of life and life satisfaction are severely impaired in patients with long-term invasive ventilation following ICU treatment and unsuccessful weaning. Ann Intensive Care 2018; 8: 38. 